\title{
Germanica
}

\section{À la dérive...}

Erika Tunner

\section{OpenEdition}

Journals

Édition électronique

URL : http://journals.openedition.org/germanica/2620

DOI : 10.4000/germanica.2620

ISSN : 2107-0784

\section{Éditeur}

Université de Lille

\section{Édition imprimée}

Date de publication : 31 décembre 1989

Pagination : 9-16

ISSN : 0984-2632

\section{Référence électronique}

Erika Tunner, «À la dérive... », Germanica [En ligne], 5 | 1989, mis en ligne le 06 février 2015, consulté le 06 octobre 2020. URL : http://journals.openedition.org/germanica/2620 ; DOI : https://doi.org/

10.4000/germanica.2620

Ce document a été généré automatiquement le 6 octobre 2020.

(c) Tous droits réservés 


\section{À la dérive...}

\section{Erika Tunner}

1 Le mal d'être est un des grands thèmes qui traversent la littérature moderne. Expression d'un sentiment de malaise individuel, il est aussi lié à des événements politiques et à des phénomènes sociaux. Il peut naître du mal métaphysique de l'imperfection, du mal physique de la souffrance, du mal moral engendré par le péché et la hantise de la culpabilité. Ce sont les trois catégories du mal selon Leibniz et on en trouve des reflets dans les études réunies dans ce cahier. De Villon à Bataille, il court, le mal ; de Grimmelshausen à Thomas Bernhard, il est présent par un vague-à-l'âme ou un ennui profond qui, selon Sainte-Beuve, a été en partie le mal du siècle. De ce mal d'être, dû souvent au désœuvrement, il est peu question chez les auteurs d'aujourd'hui. Mais il est vrai qu'eux aussi d'une sorte de désespoir avorté, d'une douleur, qui n'est pas une douleur mais qui n'est pas non plus une absence de douleur, Mißempfindungen, sensations de malaise, comme dit le héros de Martin Walser dans le roman Die Gallistl'sche Krankheit, littéralement La maladie de Gallistl, publié en France sous le titre Je ne sens pas bon. Évidemment, lorsqu'on a une santé à toute épreuve, lorsqu'on ne vit pas dans un pays qui est en guerre, lorsqu'on se voit reconnu, apprécié, lorsqu'on est capable de chasser les doutes comme des mouches importunes, tout est plus facile et le mal d'être n'est que littérature. Cependant, que la partie soit perdue depuis le début, nul ne peut le contester. Tout est vain, même si en apparence on peut croire le contraire. C'est un inconvénient qui commence avec la naissance et auquel il est bien difficile de remédier. Reste le problème du sens concret que chacun de nous essaie de donner à sa propre existence. Si ce sens est brisé, la vie est en danger, la vie se perd. Mais l'écriture est née à l'Est d'Eden... Le présent recueil se limite, par nécessité et par choix, à la littérature tout à fait contemporaine, voire actuelle.

2 À côté de quelques noms déjà bien connus du grand public, comme Ingeborg Bachmann, Christa Wolf, Franz Xaver Kroetz, Pär Lagerkvist naturellement, il y a aussi ceux qui ne sont familiers qu'à certains, tels que Rose Ausländer, Brigitte Burmeister, Marlen Haushofer, Annemarie Moser, Brigitte Schwaiger, Eva Deutsch, ou bien le grand, le très grand Hans Henny Jahnn dont la belle prose dépourvue de pathétique reste à redécouvrir. Il y a aussi des noms qui manquent et qui auraient dû trouver leur place ici. Parmi les grands absents: Thomas Bernhard dont l'œuvre entière illustre le mal d'être; de lui, il n'est 
question que d'une manière accidentelle, dans l'un ou l'autre des articles. Mais c'est un écrivain à qui beaucoup d'études ont déjà été consacrées. Il n'en est pas de même pour un compatriote de Bernhard, Gerhard Roth : chez un de ses personnages principaux, un homme muet, le mal d'être se traduit par les propos terrifiants des voix qui traversent sa tête et qui sont ses seuls interlocuteurs (Landläufiger Tod - Une mort banale). - Parmi les écrivains allemands, on aurait pu songer à Unica Zürn qui, dans son récit Dunkler Frühling (Sombre Printemps), montre magistralement le triomphe de l'écriture, si fragile qu'il soit, sur l'échec d'une vie rongée par la drogue, la maladie, la folie. Ou bien à Harmut Lange: il ne cesse de méditer sur le mal d'être, que ce soit sous forme d'un journal (Das Tagebuch eines Melancholikers - Le Journal d'un mélancolique) ou de prose narrative (Die Ermüdung - La lassitude). Ou bien à Gerhard Köpf et son roman passionnant, Innerfern, titre par lequel il faut comprendre le nom d'une localité mais aussi la perte d'une identité, la lente décomposition d'un moi qui ne cherche plus à diriger sa vie et qui cache ses angoisses sous une existence d'emprunt, impénétrable et figée. Et pourquoi ne pas avoir parlé de Monika Maron et de son livre Die Überläuferin dont la traduction vient de paraître (Le transfuge) : une femme se réveille paralysée, enfermée dans sa chambre. Les murs explosent si bien qu'elle vit des scènes " du dehors ", de la vie quotidienne, mais dans une perspective de l'imaginaire et du fantastique qui dissimule et dévoile à la fois son mal d'être. Herta Müller enfin, originaire de la Roumanie, nous rappelle que l'homme n'est qu'une proie, une bête pourchassée, qu'il n'a rien de bon à attendre ici-bas : L'homme est un grand faisan sur terre (Der Mensch ist ein großer Fasan auf der Welt).

3 Les écrivains suisses ne figurent pas dans ce recueil : en conclure que le thème du mal d'être ne se trouve pas dans leurs écrits, serait une grave erreur. Fritz Zorn, atteint d'un cancer et mort à l'âge de trente-deux ans, analyse dans Mars, avec pudeur et ironie, avec une rare pénétration aussi, les tabous et contraintes qui sont le lot de ceux qui, comme lui, sont «éduqués à la mort». Hermann Burger, avec Die künstliche Mutter ( $\mathrm{L} a$ mère artificielle), sait parler de l'effroi devant le gel de soi. Erica Pedretti enfin, place au centre de son roman Valerie oder das unerzogene Auge (Valérie ou l'œil vierge) une femme de peintre qui, au fur et à mesure que progresse sa maladie incurable, s'aperçoit qu'elle n'a existé qu'à travers le regard de son mari, qu'elle n'a pour ainsi dire jamais connu une existence propre à elle.

Ce ne sont que quelques exemples d'un inventaire qui risquerait d'être fastidieux et forcément incomplet. Nous avons seulement voulu attirer l'attention sur un certain nombre de textes où le mal d'être, d'une façon symptomatique, est au cœur du récit. Parmi les auteurs présentés, il y a une majorité de femmes; en déduire que l'expression du mal d'être soit avant tout une affaire féminine, serait se méprendre. Il suffit de songer à E.M. Cioran pour savoir qui, avec une concision admirable, exprime d'un livre à l'autre les difficultés de la condition humaine. Pour lui, le mal d'être a bien souvent son origine dans le mal qui naît du commerce avec nos semblables : «Plus nous fréquentons les hommes, plus nos pensées noircissent", dit Cioran dans un de ses Syllogismes de l'amertume, "et lorsque, pour les éclaircir, nous retournons à notre solitude, nous y trouvons l'ombre qu'elles y ont répandue ». - Comme le mal d'être est manifeste aussi bien dans les écrits en prose que dans la poésie et dans les pièces de théâtre, tous les genres ont été évoqués ici, encore que la prose, souvent autobiographique, l'emporte de loin. D'autre part, nous avons souhaité observer une certaine cohérence dans ce recueil, 
ce qui explique qu'il y a parfois des analogies dans les chemins empruntés, des parallèles dans les résultats obtenus.

$$
* * *
$$

$* *$

5 Les personnages de Franz Xaver Kroetz évoluent dans la société de la République fédérale allemande, une société où le libéralisme économique, si séduisant qu'il soit, doit remplacer bien souvent un vide idéologique et même métaphysique. Le langage n'arrive plus à articuler le malaise qui en résulte : il confine au mutisme ou verse dans un flot de paroles stéréotypées, signe de la vacuité de l'existence ou d'ententes factices et terriblement monotones. Mais Kroetz, contrairement à bien d'autres auteurs, ne sombre pas dans la résignation. Il souhaite amorcer une prise de conscience: en dépassant son égocentrisme, l'homme devrait pouvoir s'enrichir en harmonie avec son milieu. - À l'Ouest comme à l'Est, la société est faite d'hommes qui agissent uniquement selon leurs intérêts personnels et immédiats. Ce sont de grands et petits traitres aux belles manières, déclarant leur fidélité aux plus puissants. À l'Ouest comme à l'Est, une volonté féminine trop affirmée, une originalité trop marquée, s'avèrent en général être un facteur de dérangement qui, sans être d'une gravité alarmante, demande à être supprimé, par mesure de précaution. Or, la nécessité de s'adapter crée dans l'individu une dichotomie entre sa véritable nature et la fonction qu'il doit assumer. Par leur refus des compromissions qu'elles jugent indignes d'être vécues, les «héroïnes de l'absolu » de Christa Wolf n'arrivent pas à s'accomplir en tant que personne et en tant qu'artiste, et se trouvent contrariées dans leur désir de réaliser un idéal ou, tout simplement, de défendre les valeurs qu'elles ont reconnues comme justes. Mais, comme chez Kroetz, il y a chez Christa Wolf une place laissée à l'espoir : ne pouvant pas aller jusqu'au bout de leur vocation et ne choisissant pas de se renier, les protagonistes témoignent de leur malaise, de leur mal d'être dans la civilisation de progrès et de profit. Ce témoignage est à la fois un défi, un avertissement et la tentative de laisser une trace, d'échapper à l'oubli.

6 Le mal d'être évoqué par Rose Ausländer et, dans une certaine mesure, par Brigitte Schwaiger et Eva Deutsch, est quelque peu différent. Rose Ausländer, originaire d'une famille juive de la Bucovine, est un poète sans racines ou plutôt un poète aux racines errantes. Son mal d'être, pour une bonne part, est un «mal du pays». Elle vit et s'exprime dans une zone d'ombre où coexistent le souvenir et le rejet d'un passé : pendant dix ans, elle refoule l'allemand, n'écrit plus qu'en anglais. Simultanément, elle mène un combat contre l'oubli individuel et collectif, et formule un message d'humanité et de paix. Comme les personnages de Christa Wolf, elle se veut témoin et nous exhorte à témoigner à notre tour afin que la parole fixe l'éphémère aux dimensions parfois véritablement tragiques.

7 Dans le livre de Brigitte Schwaiger et d'Eva Deutsch, Die Galizianerin (La Galicienne), c'est aussi un passé douloureux, l'extermination d'une population, la destruction d'une région, la perte d'une langue (le yiddisch) qui forment le centre des réflexions, déterminent la trame de l'histoire. C'est également un livre témoignage. En utilisant une langue hybride, Eva Deutsch nous fait comprendre qu'elle fait partie de la diaspora et que cette diaspora n'a plus de langue. Sans parler d'une « diaspora intérieure » qui, de toute façon, est le lot des exilés. Mais : raconter, ne signifie-t-il pas aussi faire 
revivre - sinon vivre ? Et vivre, on le sait, c'est « s'installer » dans la précarité, il n’y a rien d'autre.

8 Chez Marlen Haushofer et chez Annemarie M. Moser, le mal d'être prend une dimension nettement pathologique. Marlen Haushofer s'inspire d'un cas de névrose où l'angoisse grandissante se propage comme les métastases d'un cancer : ce n'est pas la mort qui cause la plus grande peur, c'est le fait de vivre mal, de vivre le mal. Le « Je » dresse des barrières entre le monde et soi - un « mur invisible » qui marque à la fois un rempart et une prison, une défense et un enfermement autistique. Les personnages qui se battent contre le mal d'être ou qui l'acceptent comme une défaite dont ils ne se relèveront plus, subissent une importante modification de leur identité. Chez Marlen Haushofer, dans Eine Handvoll Leben (Une poignée de vie), l'héroïne change radicalement d'identité, simule une noyade et ne revient que des années plus tard, sous un autre nom, riche d'une expérience acquise dans un autre pays. Mais suffit-il de devenir autre, de devenir l'autre, pour échapper à un mal d'être dont les causes sont autant un malheur individuel qu'un malheur historique gravé à jamais dans notre mémoire? "Anders ", "Autre», est le nom du héros dans le roman de Brigitte Burmeister, présenté dans la rubrique «Parallèles»: Anders - différent des autres, étranger aux autres, étranger dans un milieu où il ne peut qu'être l'étranger, l'autre : une œuvre qui, par sa technique narrative, doit beaucoup au nouveau roman, mais qui est aussi éminemment politique sans nous livrer toutes les clés de ce vertigineux échange qui s'opère sans trêve entre l'imaginaire et la réalité. - Chez Anne-Marie M. Moser, une "schizophrène stabilisée " comme disent les médecins (mais qu'est-ce au juste?), les conflits sont également dus au décalage entre les aspirations intimes d'une femme et la réalité socio-culturelle franchement hostile qui l'entoure. Pour elle, ces conflits sont insolubles puisque la folie meurtrière des «sains d'esprit » apparaît comme bien plus grave que la déviance des «cas pathologiques». En fait, et la question va être posée également par Ingeborg Bachmann, personne ne sait vraiment où est la ligne de partage entre la maladie mentale, la pré-psychose, le "simple trouble psychique " et la "gale de l'âme", la dégradation des sentiments, la déshumanisation de l'être. Face à ses bourreaux, dans quel ghetto la victime peut-elle trouver refuge? Dans une secte religieuse? Dans une clinique? Dans une solitude peuplée de chimères?

9 Der Fall Franza, le «cas» Franza - Franza, un cas: Ingeborg Bachmann étudie des traumatismes psychiques successifs, un mal d'être grave et irréparable, causé par un assassinat exécuté avec raffinement et une lenteur voluptueuse. Le responsable en est le propre époux de Franza, psychiatre de surcroît, qui pratique la forme la plus dangereuse du Mal, « celle qui parvient à faire croire - et trouve commode et efficace de le croire elle-même - qu'elle défend sans faiblesse les intérêts du Bien ». Procédé plus répandu qu'on ne le pense. De tous les textes analysés dans ce cahier, ceux d'Ingeborg Bachmann sont les plus cruels et les plus sombres. En effet, si Franza, comme la plupart des héroïnes étudiées précédemment, cherche une issue dans des conduites de fuite, Ingeborg Bachmann ne laisse entrevoir aucune chance d'un lendemain possible. Le mal d'être tue. Nous pouvons toujours rêver d'une union de l'intelligence et de la sensibilité qui fera apparaitre la beauté et l'élégance de l'esprit. Bien plus souvent l'intelligence et la sensibilité se mettent au service d'un tout autre but: saisir les rênes du pouvoir, ne serait-ce que par un petit bout, acquérir « de l'importance ", et pour cela avoir de l'engagement quitte à perdre des amis dont l'inutilité se révèle affligeante pour qui veut atteindre un quelconque sommet social ou considéré comme tel. Il est dans la logique de cette entreprise d'étouffer ceux qui 
représentent une gêne, un obstacle : les écraser, les détruire à la racine, leur interdire de survivre est encore la façon la plus sûre de ses débarrasser d'eux. C'est ce que fait le docteur Jordan dont Franza est la victime. Un drame individuel et fictif, certes, mais qui permet de dégager une loi à valeur universelle : le triomphe du mal par une puissance pervertie de l'esprit.

Franza meurt. Sa souffrance et sa mort ne renvoient à rien - sinon à Rien, au Néant. Il en est de même pour Barabbas, héros du roman du même titre de Pär Lagerkvist : libéré par Pilate à la place de Jésus de Nazareth, Barabbas, homme obscur, détenu pour crime de sédition et de meurtre, subitement rendu à la vie et à l'insupportable légèreté de l'être, devient prisonnier d'un mal d'être qui l'entraîne « au bord du gouffre existentiel, du néant, du non-être ». Comme le roman d'Ingeborg Bachmann, celui de Pär Lagerkvist fait également apparaitre une loi générale illustrée par un destin personnel : c'est, de tous les textes présentés ici, celui qui proclame le plus puissamment et pardelà toutes les frontières, que la foule, par ignorance, par calcul, par méfiance d'une personnalité exceptionnelle, insaisissable, étrangère et étrange, préfère le criminel à l'innocent, préfère Barabbas à Jésus.

11 Peu connus encore, méconnus peut-être, sont en France les romans de Hans Henny Jahnn, un des génies les plus remarquables parmi les écrivains du XXe siècle, musicien, organiste et célèbre facteur d'orgues. Ses héros ne deviennent jamais "propres à l'existence».

Les échecs qu'ils ont vécus avec une acuité particulière, qu'il s'agisse d'une croyance vacillante ou d'une vocation artistique manquée les conduisent à un pessimisme noir, irréversible. Ils finissent par se sentir séparés du monde et s'identifient à un songe.

13 Avec Martin Walser nous retrouvons un écrivain de la R.F.A., mais qui dans Ressac se tourne vers un autre continent : c'est dans la Californie apparemment paradisiaque que le mal d'être s'empare d'un homme, le ronge et le perd. À qui la faute ? Lorsqu'un gibier est traqué, puis abattu, est-ce parce qu'il n'a pas été assez malin pour éviter les embûches ou parce que l'infatiguable meute des chiens a fini par avoir raison de lui ?

14 Y a-t-il une dimension positive au mal d'être? Y a-t-il une victoire sur le mal d'être? Une petite nouvelle de Pär Lagerkvist, présentée dans la rubrique «Parallèles », peut entr'ouvrir une perspective étonnante, proposer même une espèce d'antidote au mal d'être. Cependant, aucun des auteurs évoqués n'indique une transcendance. Les issues proposées sont des portes étroites dont on ne sait pas très bien sur quels chemins elles s'ouvrent. Une chose est certaine : on ne sort pas indemne du mal d'être. La vie bascule. Ce n'est pas en pure perte que le monde répand son venin sur nous. Catastrophe, bénédiction ? Les états limites ne sont-ils pas aussi les états créateurs?

\section{AUTEUR}

\section{ERIKA TUNNER}

Université Charles-de-Gaulle - Lille III 